Les cristaux coulombiens sont des structures ordonnées

\section{d'ions atomiques}

\section{ou moléculaires apparaissant}

dans des pièges à ions

à des températures proches du zéro absolu. Ces cristaux

très inhabituels représentent l'élément de base d'horloges de très grande précision,

fournissent un environnement favorable pour l'étude détaillée de réactions chimiques,

et constituent une technologie originale pour le développement d'un ordinateur quantique.

Dans cet article, nous présentons les méthodes permettant

I'obtention de tels cristaux, et nous explorons quelquesunes de leurs applications les plus spectaculaires.

\title{
Cristaux coulombiens
}

De la technologie quantique à la chimie proche du zéro absolu

Olivier Dulieu ${ }^{(1)}$ (olivier.dulieu@u-psud.fr) et Stefan Willitsch ${ }^{(2)}$ (stefan.willitsch@unibas.ch)

(1) Laboratoire Aimé Cotton, CNRS/Université Paris-Sud/ENS-Cachan, Bât. 505, Campus d’Orsay, 91405 Orsay Cedex (2) Department of Chemistry, University of Basel, Klingelbergstrasse 80, 4056 Basel, Suisse
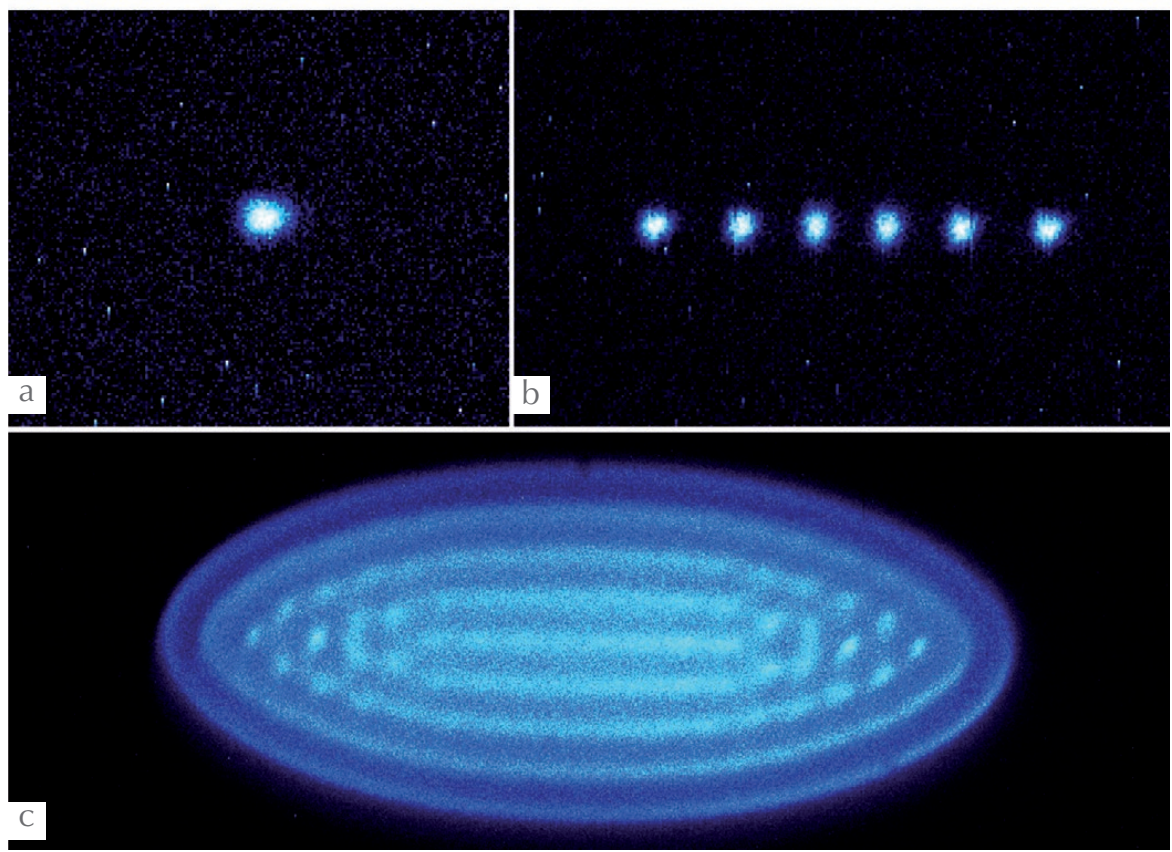

Images en fausses couleurs de plusieurs cristaux coulombiens de $\mathrm{Ca}^{+}$formés (a) d’un ion unique, (b) d'une chaîne d'ions, (c) d'un gros nuage d'ions (adaptées de Willitsch [1]). En raison de la profondeur de champ finie du dispositif d'imagerie, ces images montrent une coupe du cristal le long d'un plan central. La distance typique entre les ions est de quelques dizaines de microns.

Les progrès récents des méthodes de formation d'échantillons gazeux d'atomes et de molécules à des températures proches du zéro absolu - souvent dénommés " matière ultra-froide " - ont stimulé de nombreuses recherches, à la fois en physique et en chimie ${ }^{(\mathrm{a})}$. Les cristaux coulombiens représentent une forme particulièrement fascinante de matière ultra-froide : ce sont des structures ordonnées d'ions atomiques ou moléculaires retenus dans des pièges à ions [1]. Dans de tels systèmes, il devient possible d'observer, d'exciter et de manipuler une particule quantique unique. Ces remarquables structures quantiques ont ouvert la voie à de nombreuses applications : la mise au point d'horloges « ioniques » de très haute précision ; des mesures métrologiques permettant d'aborder des questions cruciales comme la variation dans le temps des constantes fondamentales ou l'existence du moment dipolaire électrique de l'électron ; le développement de la logique et de la simulation quantiques pour le traitement de l'information; ou encore le contrôle complet des collisions atomiques et moléculaires pour la compréhension ultime de réactions chimiques élémentaires ${ }^{(b)}$. 
$\gg>$

\section{Formation d'un cristal coulombien}

Contrairement aux cristaux ioniques bien connus de la physique du solide (comme $\mathrm{Na}^{+} \mathrm{Cl}^{-}$), les cristaux coulombiens sont composés d'ions de même charge. Leur formation comprend deux étapes essentielles : le piégeage des ions et leur refroidissement. Le piégeage par une action externe est nécessaire, puisque les ions se repoussent fortement en raison de leur charge. Un dispositif typique est le

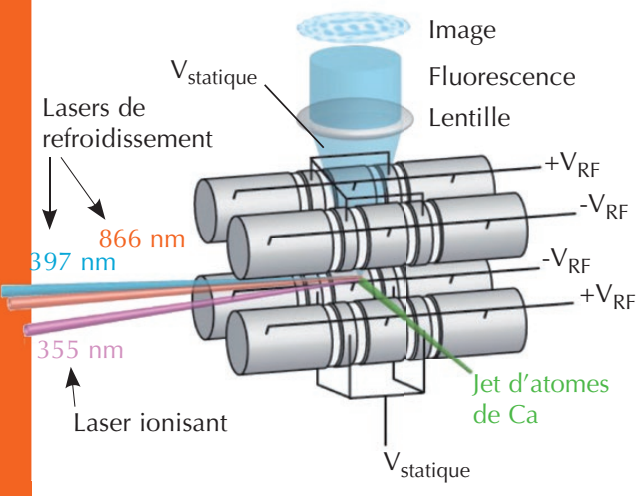

1. Schéma de principe du dispositif expérimental pour former un cristal coulombien (adapté de Willitsch et al. [3]). Des ions (ici $\mathrm{Ca}^{+}$) sont créés par ionisation d'un jet atomique (par un laser à $355 \mathrm{~nm}$ ), et sont retenus dans le piège radiofréquence formé par quatre électrodes linéaires portées par paires à des tensions $\pm V_{R F}$, dont le signe alterne dans le temps. On crée ainsi un potentiel moyen harmonique dans lequel sont retenus les ions. Deux faisceaux lasers à 397 et $866 \mathrm{~nm}$ permettent le refroidissement des ions $\mathrm{Ca}^{+}$piégés. La fluorescence générée par le refroidissement laser est recueillie par une lentille et dirigée vers une caméra. piège radiofréquence de Paul (inventé par W. Paul), consistant le plus souvent en un ensemble d'électrodes linéaires recevant des tensions statiques et variables (fig. 1). Une autre option - moins fréquente dans ce contexte - est l'utilisation d'un piège de Penning, où se superposent un champ magnétique et un champ électrique statiques [2]. La formation d'un cristal coulombien - c'est-à-dire la mise en ordre des ions dans la zone confinée du piège - ne peut avoir lieu que si l'énergie potentielle des ions excède largement (par un facteur $\approx 170$ ) leur énergie cinétique. Cela nécessite qu'un gaz de particules légères comme les ions atomiques soit refroidi à une température de l'ordre de quelques millièmes de degrés au-dessus du zéro absolu (quelques millikelvins) ${ }^{(c)}$. Ce régime peut être atteint au moyen du refroidissement laser, consistant à diminuer la vitesse des particules par absorptions répétées de photons d'un laser. Mais cette approche est limitée à des ions atomiques de structure simple, comme les ions alcalinoterreux $\left(\mathrm{Mg}^{+}, \mathrm{Ca}^{+}, \mathrm{Sr}^{+}, \mathrm{Ba}^{+}\right)$avec un électron de valence.

Les cristaux coulombiens apparaissent alors sous plusieurs formes dans un piège de Paul (figure de la p. 91) : (a) un ion isolé, (b) une chaîne d'ions, ou encore (c) un gros cristal macroscopique contenant des centaines d'ions, et dont la taille peut atteindre une fraction de millimètre ! La forme sphéroïdale du gros cristal provient $\mathrm{du}$ profil harmonique du potentiel de piégeage. Ce cristal coulombien n'a donc pas de symétrie de translation.

\section{Les cristaux coulombiens moléculaires}

Au contraire des atomes, les molécules ne peuvent pas être refroidies par laser (à part quelques rares exceptions) en raison de la structure complexe de leur spectre de niveaux d'énergie, qui rend impossible l'absorption répétée de photons du laser. Cependant, une méthode originale a été employée pour refroidir des ions moléculaires à des températures similaires à celle des ions atomiques : le refroidissement "sympathique ", dont le principe est schématisé sur la figure 2a. Les ions moléculaires piégés échangent de l'énergie cinétique par collision avec les ions atomiques, énergie qui est finalement dissipée par le refroidissement laser. Un cristal coulombien à deux composantes est ainsi créé, dans lequel seuls les ions atomiques sont fluorescents, tandis que les ions moléculaires ne sont pas directement visibles. La comparaison de l'image observée avec une image simulée, produite par une méthode de dynamique moléculaire, permet de rendre ces ions artificiellement visibles (fig. 2 b).

Un des principaux intérêts des cristaux coulombiens est le haut niveau de contrôle des particules piégées. Le refroidissement gèle leur mouvement et les fixe dans l'espace, tandis que leur état quantique interne peut être préparé et manipulé par des lasers. Ce dernier point est établi depuis longtemps pour des ions atomiques, qui ne présentent que peu de degrés de liberté interne liés aux
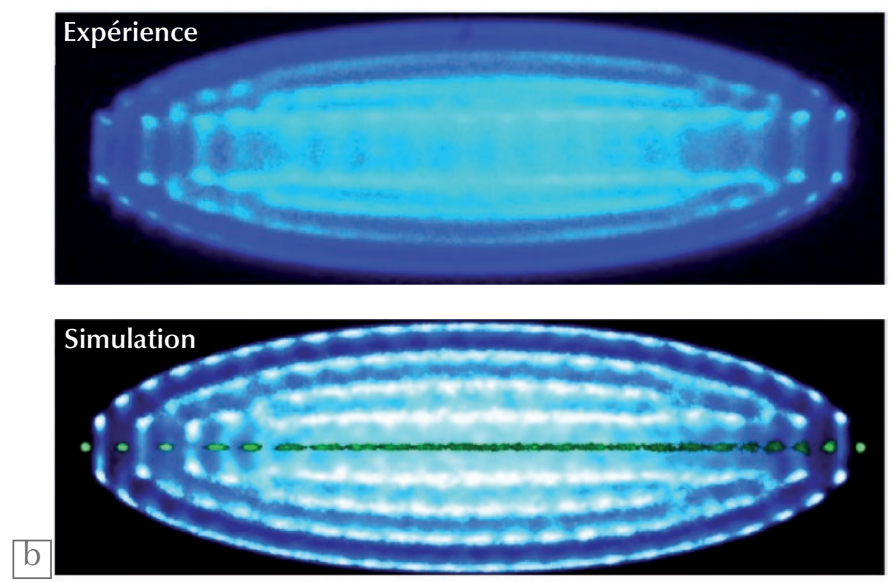

2. Refroidissement sympathique (adapté de Willitsch [1]).

(a) Principe : des ions moléculaires piégés (en vert) sont refroidis par collision avec des ions atomiques (en orange) piégés et refroidis par laser. II en résulte la création d'un cristal coulombien à deux composantes, comme celui des photos b.

(b) En haut : image d'un cristal contenant 25 ions $\mathrm{N}_{2}^{+}$(localisés dans la partie centrale non fluorescente du cristal), sympathiquement refroidis par 925 ions $\mathrm{Ca}^{+}$. En bas : simulation de dynamique moléculaire, où les ions $\mathrm{N}_{2}{ }^{+}$sont représentés en vert. 


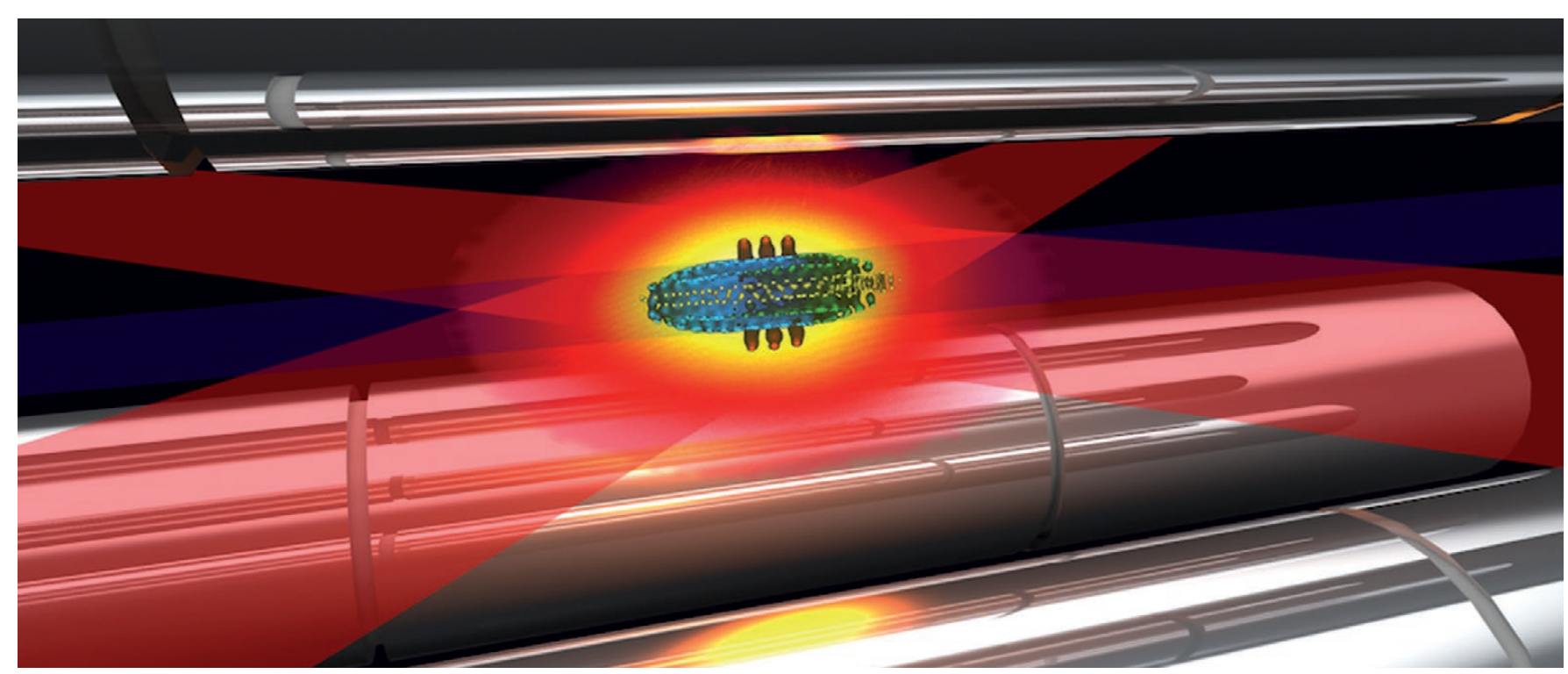

3. Réaction chimique froide. Illustration d'un cristal coulombien d'ions $\mathrm{Ba}^{+}$(en bleu) immergé dans un nuage d'atomes ultra-froids de rubidium (Rb, tache jaune), lui-même retenu dans un piège magnéto-optique (symbolisé par des faisceaux laser rouges). Les réactions chimiques froides conduisent au remplacement progressif d'ions $\mathrm{Ba}^{+}$par des produits ioniques atomiques $\left(\mathrm{Rb}^{+}\right)$et moléculaires $\left(\mathrm{RbBa}^{+}\right)$, représentés en jaune au centre et en rouge en périphérie.

électrons et aux spins nucléaires. En revanche, les ions moléculaires présentent des degrés de liberté supplémentaires liés à leurs mouvements de vibration et de rotation qui rendent ce contrôle moins immédiat. La mise au point de méthodes permettant la manipulation et le contrôle des états quantiques internes des ions moléculaires dans un cristal coulombien est, depuis plusieurs années, l'objectif majeur de ce domaine, qui a vu des premières réalisations très récemment [1].

\section{Les applications : de la logique quantique...}

C'est avec des ions atomiques piégés que le refroidissement laser a été démontré pour la première fois dans les années 1970 [4]. Mais l'avancée décisive dans ce domaine est intervenue lorsque Ignacio Cirac et Peter Zoller ont réalisé qu'une chaîne d'ions " cristallisés " pouvait constituer l'élément de base pour un ordinateur quantique [5]. Depuis, les développements expérimentaux basés sur des cristaux coulombiens ont conduit à une des réalisations actuelles les plus élaborées d'un système d'information quantique [6]. Ce qu'il convient maintenant d'appeler la simulation quantique est aussi devenue une application importante : la dynamique de l'hamiltonien d'un système complexe - par exemple issu de la matière condensée, dont les paramètres sont difficilement ajustables en laboratoire - est simulée au travers de son implémentation dans un autre système comme un cristal coulombien : on montre que le système obtenu est alors mathématiquement équivalent, tout en bénéficiant des possibilités de manipulation du cristal coulombien.

\section{... et des mesures de précision...}

La possibilité d'isoler des particules uniques dans l'environnement ultravide très contrôlé que constitue un piège à ions, et de maîtriser leur mouvement et leur état quantique interne, a permis le développement d'une nouvelle génération d'horloges atomiques qui figurent actuellement parmi les plus précises [7]. Le principe d'une telle horloge repose sur la mesure de la fréquence d'une transition électronique d'ion piégé unique, le plus souvent situé dans le domaine visible ou ultraviolet. Cela permet d'atteindre une précision de la mesure qui surpasse celle réalisée par une horloge atomique conventionnelle, qui implique une transition dans le domaine des radiofréquences. L'utilisation d'un ion moléculaire piégé comme $\mathrm{H}_{2}{ }^{+}$est même envisagée pour réaliser une horloge de grande précision [8]. En parallèle, cette précision extrême des mesures sur des ions piégés permet d'aborder des questions fondamentales habituellement dévolues à la physique des hautes énergies. Par exemple, plusieurs projets s'intéressent à la variation sur de grandes échelles de temps des constantes fondamentales comme la constante de structure fine ou le rapport des masses de l'électron et du proton, ou encore à l'influence de la gravitation sur les fréquences de transitions atomiques [9].

\section{... à la chimie ultra-froide contrôlée}

Les chimistes ont aussi commencé à s'intéresser aux cristaux coulombiens pour le refroidissement sympathique d'espèces moléculaires ioniques, permettant l'étude détaillée de réactions chimiques entre espèces neutres et ioniques, et à très basse température. Plusieurs types d'expériences ont été récemment développées, combinant des pièges à ions avec des sources d'atomes froids [10,11] ou de molécules froides [12] (fig. 3). Des processus chimiques inhabituels peuvent être étudiés en régime ultra-froid et en phase très diluée, comme par exemple la formation d'ions moléculaires par émission d'un photon au cours de la collision entre l'atome et l'ion atomique. Au-delà de l'environnement artificiel fourni par ces expériences, ce type de processus joue un rôle fondamental dans la compréhension de la chimie de l'espace interstellaire. La dynamique de la collision ultra-froide entre trois particules, cruciale dans un milieu dense comme un condensat de Bose-Einstein, a pu être explorée [10], de même que les subtiles interactions à grande distance qui ne se manifestent qu'en régime ultra-froid sans être masquées par l'agitation thermique des particules [11]. De façon générale, de telles conditions 


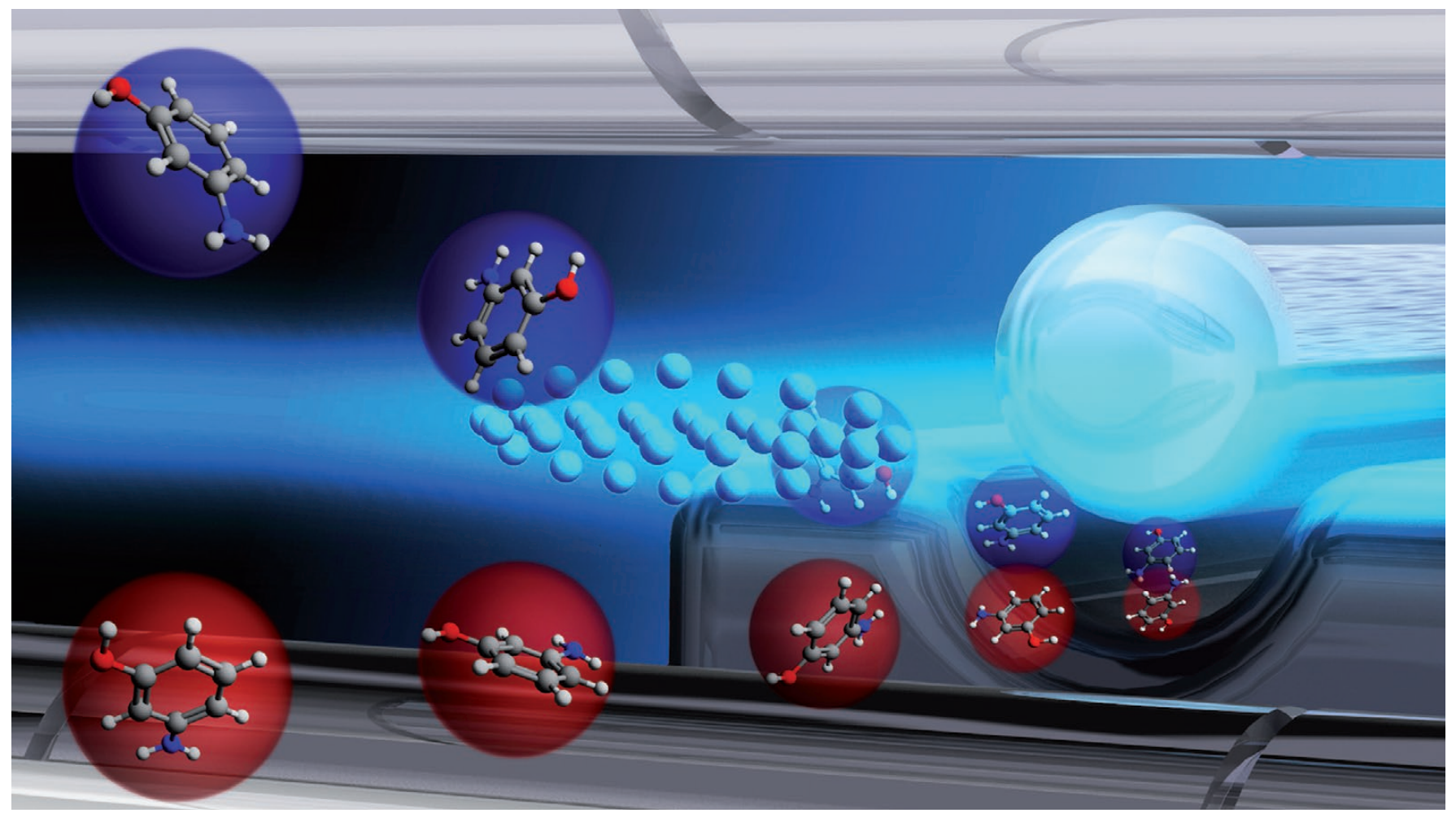

4. Illustration d'une expérience de chimie froide contrôlée. Deux conformations différentes (soulignées en rouge et en bleu) de molécules organiques «3-aminophénol » issues d'un même jet moléculaire sont séparées spatialement par leur interaction avec un champ électrique inhomogène (figuré par l'électrode à droite de la figure), grâce à la différence de la valeur de leurs moments dipolaires électriques. Cela correspond à une version électrostatique moléculaire de la célèbre expérience de Stern et Gerlach (1922), dans laquelle les atomes ont été séparés spatialement par un champ magnétique, conduisant à la découverte de leur spin. Les molécules peuvent ainsi être dirigées sélectivement vers la cible constituée par un cristal coulombien d’ions $\mathrm{Ca}^{+}$: la conformation " bleue » est envoyée vers le cristal, tandis que la conformation rouge l'évite. On étudie ainsi l'influence de la forme de la molécule sur le déroulement de sa réaction chimique avec les ions piégés. (IIlustration de Y.-P. Chang, DESY [13].)

\section{$\gg>$}

permettent de mieux comprendre les détails des processus chimiques, grâce à leur observation dans des conditions très contrôlées.

Les cristaux coulombiens sont aussi mis à profit pour l'étude détaillée de la dynamique de molécules complexes. Par exemple, de nombreuses molécules polyatomiques dans leur état fondamental présentent plusieurs formes stables, appelées conformations, qui ne different que par la rotation d'une ou plusieurs parties de la molécule autour de l'axe de la liaison chimique. L'influence de ces conformations sur une réaction chimique est une question ouverte depuis longtemps, mais difficilement accessible car aux températures usuelles, l'agitation thermique entraine des changements de conformation. La figure 4 illustre une expérience dans laquelle des molécules polyatomiques sont préparées dans une conformation donnée, et y subsistent grâce à une méthode de refroidissement adiabatique. Elles peuvent ainsi interagir sélectivement avec une cible constituée par un cristal coulombien lui aussi très froid, et révéler les différences de réactivité induites par leur forme [13].

\section{Conclusion}

Avec ces progrès récents spectaculaires, la technologie des cristaux coulombiens atomiques et moléculaires a permis l'émergence d'un domaine scientifique situé à l'interface de la physique quantique, de la physique atomique et moléculaire, et de la chimie. De nouvelles perspectives s'ouvrent en direction de l'amélioration des performances des horloges atomiques qui fera reculer les limites de la précision des mesures de fréquences, du développement d'une ingénierie quantique de molécules uniques qui étend et augmente les possibilités déjà explorées avec les atomes, et de la découverte de nouvelles méthodes pour l'étude détaillée et le contrôle de réactions chimiques. Sans exagération, la recherche sur et avec les ions ultra-froids est devenue un domaine brûlant!

\section{Références}

1• S. Willitsch, Int. Rev. Phys. Chem. 31 (2012) 175.

2• R.C. Thomson et al., J. Phys. B 42 (2009) 154003.

3• S. Willitsch et al., Phys. Chem. Chem. Phys. 10 (2008) 7200

4• D.J. Wineland et al., Phys. Rev. Lett. 40 (1978) 1639.

5• J.I. Cirac et P. Zoller, Phys. Rev. Lett. 74 (1995) 4091

6• H. Häffner et al., Phys. Rep. 469 (2008) 155.

7• A.D. Ludlow et al., arXiv:1407.3493v2 [physics. atom-ph] (2014).

8• S. Schiller et al., Phys. Rev. Lett. 113 (2014) 023004.

9• C.W. Chou et al., Science 329 (2010) 1630.

10• A. Härter et J. Hecker Denschlag, Contemp. Phys. 55 (2014) 33.

11• S. Willitsch, Proc. Int. Sch. Phys. Enrico Fermi 189 (2015) 255.

12• S. Willitsch et al., Phys. Rev. Lett. 100 (2008) 043203. 13• Y.P. Chang et al., Science 342 (2013) 98.

(a) Ce domaine a été récompensé par deux prix Nobel de physique, en 1997 et 2001.

(b) Ces avancées spectaculaires ont également été reconnues par l'attribution du prix Nobel de physique 2012 à D. Wineland (conjointement à Serge Haroche).

(c) Notons qu'une telle configuration peut être réalisée à température ambiante avec des particules plus lourdes, comme des grains de poussières. 